\title{
Assessment of blood vessel mimics with optical coherence tomography
}

\author{
Garret T. Bonnema, The University of Arizona College of Optical Sciences, Tucson, Arizona 85721 \\ Kristen O. Cardinal, The University of Arizona Division of Biomedical Engineering, Tucson, Arizona \\ 85721 \\ James B. McNally, The University of Arizona College of Optical Sciences, Tucson, Arizona 85721 \\ Stuart K. Williams, The University of Arizona Division of Biomedical Engineering, Tucson, Arizona \\ 85721
}

Jennifer K. Barton, The University of Arizona College of Optical Sciences and Division of Biomedical Engineering, Tucson, Arizona 85721

\begin{abstract}
Optical coherence tomography (OCT) is an imaging modality that enables assessment of tissue structural characteristics. Studies have indicated that OCT is a useful method to assess both blood vessel morphology and the response of a vessel to a deployed stent. We evaluated the ability of OCT to visualize the cellular lining of a tissue-engineered blood vessel mimic (BVM) and the response of this lining to a bare metal stent. We develop a side-firing endoscope that obtains intraluminal, longitudinal scans within the sterile bioreactor environment, enabling time-serial assessment. Seventeen BVMs are imaged with the endoscopic OCT system. The BVMs are then evaluated via fluorescence microscopy and/or standard histologic techniques. We determine that (1) the OCT endoscope can be repeatedly inserted without visible damage to the BVM cellular lining, (2) OCT provides a precise measure of cellular lining thickness with good correlation to measurements obtained from histological sections, and (3) OCT is capable of monitoring the accumulation of cellular material in response to a metallic stent. Our studies indicate that OCT is a useful technique for monitoring the BVM cellular lining, and that OCT may facilitate the use of BVMs for early stage device assessment.
\end{abstract}

Keywords: optical coherence tomography; endoscope; tissue engineering; blood vessel mimic; stents. 


\section{Introduction}

\subsection{Optical Coherence Tomography in Vascular Imaging}

Optical coherence tomography (OCT) is a tissue-imaging modality that enables longitudinal and crosssectional assessment of tissue structure. OCT is analogous to ultrasound, except that near-IR light is used rather than sound, and signal is generated at index of refraction mismatches rather than acoustic index mismatches. With an axial resolution of approximately $10 \mu \mathrm{m}$, OCT provides an order of magnitude resolution improvement with a greater dynamic range as compared to commercially available intravascular ultrasound systems. ${ }^{1,2}$ The major trade-off accompanying these benefits is the smaller depth of penetration; OCT can image approximately 1 to $2 \mathrm{~mm}$ beneath the surface of typical tissues.

Several studies have been performed evaluating OCT's ability to assess blood vessel morphology and pathology. OCT has been used to evaluate the structural characteristics of the arterial wall, including thickness measurements of key arterial wall components such as the intimal and medial layers. ${ }^{3,4}$ The structural information provides a valuable tool in assessing different forms of atherosclerotic plaques, including the thin-cap fibroatheroma. ${ }^{1,5}$ Several researchers have used OCT to evaluate the deployment of coronary stents and the vascular response to these implants. OCT has been used to monitor stent deployment characteristics such as prolapse, ${ }^{6-8}$ dissection,,${ }^{6,7}$ apposition, ${ }^{6,7}$ and recoil. ${ }^{9}$ Researchers have noted that OCT can be used to monitor the presence of a neointimal layer ${ }^{6,10-13}$ and thrombus ${ }^{14}$ in stented arteries. These studies demonstrate the utility of OCT for evaluating both native blood vessels and the tissue response to vascular interventions.

\subsection{OCT in Vascular Implant Development}

Although metal stents significantly reduce the occurrence of immediate vessel recoil following percutaneous transluminal coronary angioplasty, studies have shown that approximately 10 to $20 \%$ of patients require a secondary procedure due to restenosis. ${ }^{15-17}$ The rate of failure increases for high-risk populations such as diabetics. ${ }^{17,18}$ Advances are being made to reduce the incidence of in-stent restenosis, most notably drug- eluting stents. ${ }^{17}$ Additional research is being performed in the development and evaluation of bioresorbable stents. ${ }^{19-21}$

To evaluate these new stent technologies, an assessment of the tissue response to the stent is required. The development of novel stent designs is prolonged by expensive and time- consuming animal and clinical trials. Moreover, the lack of an intravital, high-resolution imaging system to evaluate in-stent tissue responses results in limited temporal endpoints. Current technologies require sacrifice of the animal to retrieve the stent. Novel in vitro models are under development to provide rapid assessment of cellular responses to stents. As described by Cardinal et al., a blood vessel mimic (BVM) may serve as a system to model a human artery in vitro. ${ }^{22}$ Together with the minimally invasive, high-resolution visualization capabilities of OCT, the BVM has the potential to enable rapid and inexpensive evaluation of tissue response to various stent designs and drug coatings.

A current problem associated with drug-eluting stents is the eventual development of a thrombus due to the presence of the nonendothelialized metallic stent. Although this in vitro system does not have the blood components necessary for a study of thrombogenesis, the BVM models the intimal response. Therefore, OCT can visualize the progression, (or lack thereof), of this antithrombogenic tissue lining.

There has been limited previous research as to the utility of OCT for imaging tissue engineered constructs. Yang et al.23 imaged poly-l-lactic acid scaffolds sodded with MG63 bone cells. These constructs were removed from the culturing system, rinsed with saline, and then imaged with OCT. We have previously imaged a vascular construct consisting of smooth muscle cells sodded onto the luminal surface of a polyurethane scaffold. This construct was removed from its bioreactor and imaged while 
submerged in both saline and anticoagulated blood.24 Mason et al. visualized an artificial vessel constructed of human mesenchymal stem cell/alginate hydro- gel within a bioreactor, indicating that imaging grafts in a bioreactor is feasible. 25 However, none of these studies examined the utility of OCT for quantifying the development and response of a luminal cellular lining within the sterile envi-ronment of a bioreactor system.

In this paper, we evaluate the use of OCT to visualize the cellular lining of a human BVM and the response of this lining following implantation of a stent. The aims of this study were to evaluate the feasibility of (1) introducing the OCT endoscope in a repeated, sterile fashion to obtain images without damaging the BVM; (2) obtaining accurate measurements of cellular lining thickness; and (3) visualizing the cellular lining response to bare metal stents.

\section{Materials and Methods}

\subsection{OCT System}

Figure 1 presents a schematic of the OCT system. The source is a 1300-nm superluminescent diode (SLD) with a 100-nm bandwidth (SuperLum D1300-HP, Moscow, Russia), providing a measured axial resolution of $12 \mu \mathrm{m}$ in air. The source was split into the sample and reference arms using a 50:50 fiber coupler. The power on the sample was measured to be $1.2 \mathrm{~mW}$. The reference arm pathlength was modulated with the use of a rapid scanning optical delay line. 26 This setup was capable of producing ascan rates from 100 to 1000 a-scans/s, which were sufficient for imaging within these in vitro constructs. The interferometric signal was detected using a photodiode (Optiphase Model V500, Van Nuys, California) and demodulated using custom logarithmic amplifying circuitry. The signal was acquired and processed using custom C\# software with a high-speed National Instruments data acquisition board (PCI6111, National Instruments, Austin, Texas).

The sample arm of the interferometer was a miniature endoscope containing one OCT channel (singlemode fiber) and three accessory channels $(200-\mu \mathrm{m}$, multimode fibers). These accessory channels can be used for other imaging needs such as the insertion of an aiming beam or laser-induced fluorescence measurements. A similar endoscope design has been described previously.27 Light in the OCT channel was focused by a gradient-index (GRIN) lens, producing a lateral resolu-tion of $20 \mu \mathrm{m}$ at a working distance of $400 \mu \mathrm{m}$ outside the endoscopic window. A rod prism was used to direct the light out of the side of the endoscope window. Instead of using the standard 45-deg angle, the current rod prism was designed to have a 48-deg angle to reduce back-reflections from the glass endoscope window. The endoscopic optics such as the GRIN lens and rod prism were housed within a 2-mm-outer-diam, 40-mmlong quartz optical window. Two stepper motors were used to rotate and translate the optics within the optical window, providing the means to image anywhere within the construct. The motion was transferred to the optics using an inner polyimide tubing, while the endoscopic window remained fixed in place by the outer sheath of the endoscope.

An optional 20 -cm-long quartz tube was used to provide a sterile cover for the endoscope. The $2.5-\mathrm{mm}$ outer-diam, 2.2-mm-inner-diam windows InnovoQuartz were easily sterilized and were mechanically robust due to their small size. A small amount of index-matching fluid was placed between the endoscope and the sterile window to reduce the reflections from the glass-air interfaces. Ethanol was typically used due to the low surface tension and slightly greater refractive index than water.

\subsection{Development and Imaging of the BVM}

The development of the BVM required three major components: a thin cellular lining, a scaffold supporting that lining, and a bioreactor system that provided nutrient rich media to the developing 
construct. 22 Many types of materials can be used as the synthetic scaffold including expanded polytetrafluoroethylene, Dacron ${ }^{\circledR}$, polypropylene, and polyurethane. 28 For these mimics, the tubular scaffold was made of the biocompatible, nondegradable expanded polytetrafluoroethylene (ePTFE). This common scaffold material is used clinically in large-diameter bypass grafts, and extensive research has been performed with ePTFE regarding modification techniques and endothelial cell growth and adhesion. Human microvessel endothelial cells were isolated from adipose tissue and pressure-sodded onto the luminal surface of the ePTFE. This procedure caused the formation of a thin, confluent cellular lining on the lumen of the scaffold. The construct was cultivated in the bioreactor for 14 to 21 days prior to imaging.

All imaging occurred within the BVM chamber of the bioreactor system. Figure 2 shows schematics of the bioreactor and BVM chamber. The vertically-oriented BVM remained completely submerged in the bioreactor media. The endoscope was inserted into the plastic tubing of the bioreactor and fed into the BVM via a plastic barbed fitting. Once the endoscopic optics were adjacent to the distal connector, longitudinal scans were acquired by translating the optics in the proximal direction through the BVM. The OCT images were displayed such that the luminal surface, and thus the cellular lining, was located toward the top of the image. The length of each OCT image was $15 \mathrm{~mm}$ and the depth was $2 \mathrm{~mm}$, assuming an average sample index of refraction of 1.33. Each image consisted of 3000 pixels in the longitudinal direction and 625 pixels in the depth direction and took approximately $15 \mathrm{~s}$ to acquire.

\subsection{Experiments}

Three sets of experiments with the BVMs were performed. The first experiment demonstrated the feasibility of imaging within a BVM without damage due to endoscopic insertion. Four BVMs were developed under identical protocols for 21 days. 22 Two BVMs were fixed immediately in Histo-choice, and two BVMs were imaged with the endoscopic OCT system prior to fixation. Longitudinal scans were obtained at four equally spaced angular positions within the mimic. Histological cross sections using standard paraffin embedding and hematoxylin and eosin (H\&E) staining were prepared on all BVMs.

The second experiment assessed the ability of OCT to characterize the BVM cellular lining within a bioreactor. To observe a variety of lining thicknesses, 12 BVMs were sodded with a range of initial cellular densities ( 2 X 105 to 8 X 105 cells $/ \mathrm{cm} 2)$. The vessels were allowed to develop in the bioreactor under flow for 14 days. 22 The BVMS were then imaged within the bioreactor using the endoscopic OCT system. Longitudinal scans were obtained at eight equally spaced angular positions. In addition to the equal angularly spaced images, an image was acquired over a longitudinal blue line painted onto the abluminal side of the vessel scaffold during manufacturing. After imaging, the BVMs were immediately fixed in Histochoice and histology was prepared as already describe, except that the sample was oriented longitudinally. The slice that passed through the centerline of the BVM and contained the longitudinal blue line was selected and compared to the OCT image taken at the angular position of the blue line.

The thickness of the cellular lining was measured for all 12 vessels. A comparison was performed between OCT images and corresponding histological sections using statistical measures collected over the total length of the image. To facilitate these measurements, a MATLAB® (Mathworks, Version 7.0) program was developed that enabled the operator to specify two cubic spline curves identifying the luminal and abluminal surfaces of the cellular lining. The mean, median, and stan-dard deviation of the vertical separation of the curves were calculated. For thickness measurements in OCT, the index of refraction of the cellular lining was assumed to be 1.352, corresponding to a measurement of the intima, as reported by van der Meer et al.29

The third experiment evaluated whether OCT could visualize the tissue response to a bare metal stent. One BVM was developed for 14 days. Before deployment of the stent, the BVM was imaged within the bioreactor to ensure a confluent lining of cells existed on the luminal surface of the ePTFE. A bare metal 
stent was then deployed in the BVM using a balloon catheter. The cellular response was imaged at 3, 7, and 14 days after stent deployment within the bioreactor using the same imaging protocol as the previously described experiment.

\section{Results and Discussion}

\subsection{Robustness Study}

Two specific criteria were defined for a healthy, undisturbed cellular lining. These criteria were based on prior experience with BVM development and confirmed with the two BVMs that were immediately fixed without imaging. First, the histology of the BVM should show no bare areas, which would indicate that insertion or removal of the endoscope sheared off cells from the underlying ePTFE. On inspection of the cross- sectional histology of the two BVMs imaged with OCT, no suspected regions of cellular removal could be identified. The second criterion stated that the cellular lining should be firmly and continuously attached to the underlying ePTFE. A sepa-ration of the cells from the ePTFE could suggest that the endoscope had induced enough stress to weaken the interface between these two layers. Histology of the two BVMs imaged with the endoscope showed a cellular lining that was firmly attached to the underlying ePTFE. Overall, no anomalous features could be identified to indicate that imaging had damaged the cellular lining. These experiments suggest that with our imaging equipment and protocol, endoscopic imaging can be performed without visible damage or disruption to the cellular lining.

\subsection{Cellular Lining Measurement Study}

OCT images and histology slides were obtained from all 12 BVMs. Figure 3 shows an example OCT image/light micro-graph pair. The cellular lining is observed by OCT as a hy-pointense layer with an uneven luminal surface. The underlying scaffold is visualized as a hyperintense region. A distinct, smooth interface between the cellular lining and the scaffold can be seen in Fig. 3. The bioreactor media is transparent to the 1300-nm OCT light and thus is represented by the dark area above the cellular lining.

The cellular lining morphology of the BVM can be modulated by adjusting developmental parameters such as the cellular sodding density. This provides opportunities to form unique, uneven cellular lining structures within the BVM. In Fig. 4 an OCT image and a light micrograph of the corresponding histological section are shown from a BVM that contained a cellular lining characterized by islands of cells congregating on the surface of the ePTFE. A high degree of correlation can be seen between these two types of images.

The accurate visualization of this cellular lining reinforces the utility of OCT as a tool for on-going assessment. Currently, the BVMs are developed without directly monitoring the cellular lining. The endoscopic OCT system provides a minimally disruptive technique to observe the distribution and maturation of the cellular lining.

The thickness of the cellular lining was measured from the OCT images. The minimum cellular lining thickness that could be detected by the OCT system was empirically found to be approximately $25 \mu \mathrm{m}$, more than two times larger than the axial resolution of the OCT light source. The ability of OCT to resolve the cellular lining was limited by the small difference in backscattered intensity between the cellular lining and scaffold. We also noted that islands of cells were easier to identify than a thin confluent cellular lining.

A comparison between the average thickness measured with OCT and histology for each BVM can be seen in Fig. 5. The error bars are the standard deviation of the thickness measurements. Linear regression was performed on the data and a reasonable correlation was found with an R2 value of 0.71 . While every 
attempt was made to register OCT and histological images using the blue line on the ePTFE as a fiducial, it is unlikely that the approximately 20 - $\mu \mathrm{m}$-thick OCT image and the $6-\mu \mathrm{m}$-thick histological section were exactly coaligned, which may be the major source of error. All measurement data would lie on a fit line with a slope of unity if histology exactly correlated to OCT. In this case, the slope was slightly greater than unity, indicating that OCT measured on average a 19\% thicker layer than histology. This finding is consistent with the understanding that tissue preparation in the histological process causes shrinkage of the tissue. This difference of structural thickness measurements has been previously reported in OCT-histology comparisons of arterial wall components.4

\subsection{Response to a Metallic Stent}

Figure 6 displays a series of OCT images from the stented BVM. In this vessel, the prestent image in Fig. 6(A) has a thin cellular lining on the luminal surface of the ePTFE. The cellular lining was thinner and more hyperintense than the 12 unstented BVMs. These differences are thought to be due to the lower sodding density and different flow conditions for this particular vessel. Based on previous experience with BVM development, lower sodding density, on average, results in a thinner cellular lining. The increased cellular density of this thinner lining could explain the more hyperintense presentation of the cellular lining in Fig. 6(A).

After deployment, OCT visualized several features previously observed in studies of stented arteries. The stent struts were represented as bright horizontal lines with dark shadows underneath, consistent with previous studies involving stented human arteries.6-14 Occasional bright vertical streaks occurring near the struts are artifacts due to the strong reflection saturating the detection electronics. The typical strut width for the implanted stent was measured to be $80 \mu \mathrm{m}$ wide with a bright-field microscope. However, the OCT imaging plane rarely intersects the stent struts perpendicularly, and may intersect the stent at a cross-member or strut branch point. Consequently, the reflections from the stent struts may be up to 750 $\mu \mathrm{m}$ wide. Figure 6(B) shows a small amount of cellular material that was separated from the underlying ePTFE due to the deployment of the stent. While the BVM is obviously different in composition from atherosclerotic human arteries, other researchers have noted damage following stent deploy- ment such as intimal and medial vessel wall dissection. In this study, OCT also provided an accurate visualization of stent apposition to the BVM wall. Apposition characteristics of stented arteries have been observed using OCT by other researchers.6,7,13

At 3 days postdeployment, OCT images showed the presence of additional material around the sides of the stent struts. The optical characteristics of this material were consistent with cellular lining. In the series of images acquired 3 days postdeployment, significant accumulation was visualized only along a few isolated stent struts, as shown in Fig. 6(C). At 7 days postdeployment, approximately half of the stent struts had associated areas of visible tissue formation. Accumulation near 2 of the 5 imaged stent struts can be seen in Fig. 6(D), where the most significant areas of development are indicated with white arrows. As seen in Fig. 6(E), at 14 days postdeployment the stent struts were uniformly covered by the cellular lining. The presence of cells in this tissue was later confirmed using a nuclear-specific fluorescent stain (bisbenzimide) and epifluorescence microscopic observation.22 This finding suggests that OCT imaging enables time-serial assessment of tissue response in association with stent struts. Although the cellular response of the stented BVM is not equivalent to stented human arteries, the two share a similar feature of the presence of cellular accumulation on the stent struts. 5,6,11-13

The epifluorescence analysis with bis-benzimide-stained BVMs visualized a monolayer of cells on the stent struts at the 14-day time point. This monolayer was not detected in the corresponding OCT images. The thinness of the layer in this case precluded visualization, however, specular reflection from stent struts will diminish the ability to measure larger overlying cell linings. Frequently, stent struts reflected 
enough light to saturate the OCT system, as evidenced by vertical streaking [for example, in the enlarged portion of Fig. 6(B)]. Occasionally, saturation of the detection electronics occurred across the entire strut, increasing the full width half maximum of the axial point spread function from the surface of the strut on the order of $50 \mu \mathrm{m}$. This saturation may place a lower limit on the cell layer thickness that can be measured. To reduce the complications introduced by this saturation artifact, the endoscope was designed such that the light exited the endoscope slightly off angle ( $7.5 \mathrm{deg}$ in water) to suppress the specular reflections from the stent struts as well as the endoscope window.

\section{Conclusions}

Our results suggest that OCT can be a valuable tool in (1) monitoring the development of the BVM cellular lining and (2) evaluating the cellular response to bare metal stents. Without contamination or physical disruption to the cellular lining, OCT provided thickness measurements that were consistent with those seen in corresponding histological sections, and visualized cellular accumulation near the struts of the metal stent. Future studies will assess the potential for using OCT to evaluate experimental stent designs in the novel BVM model system. 


\section{Figure 1}

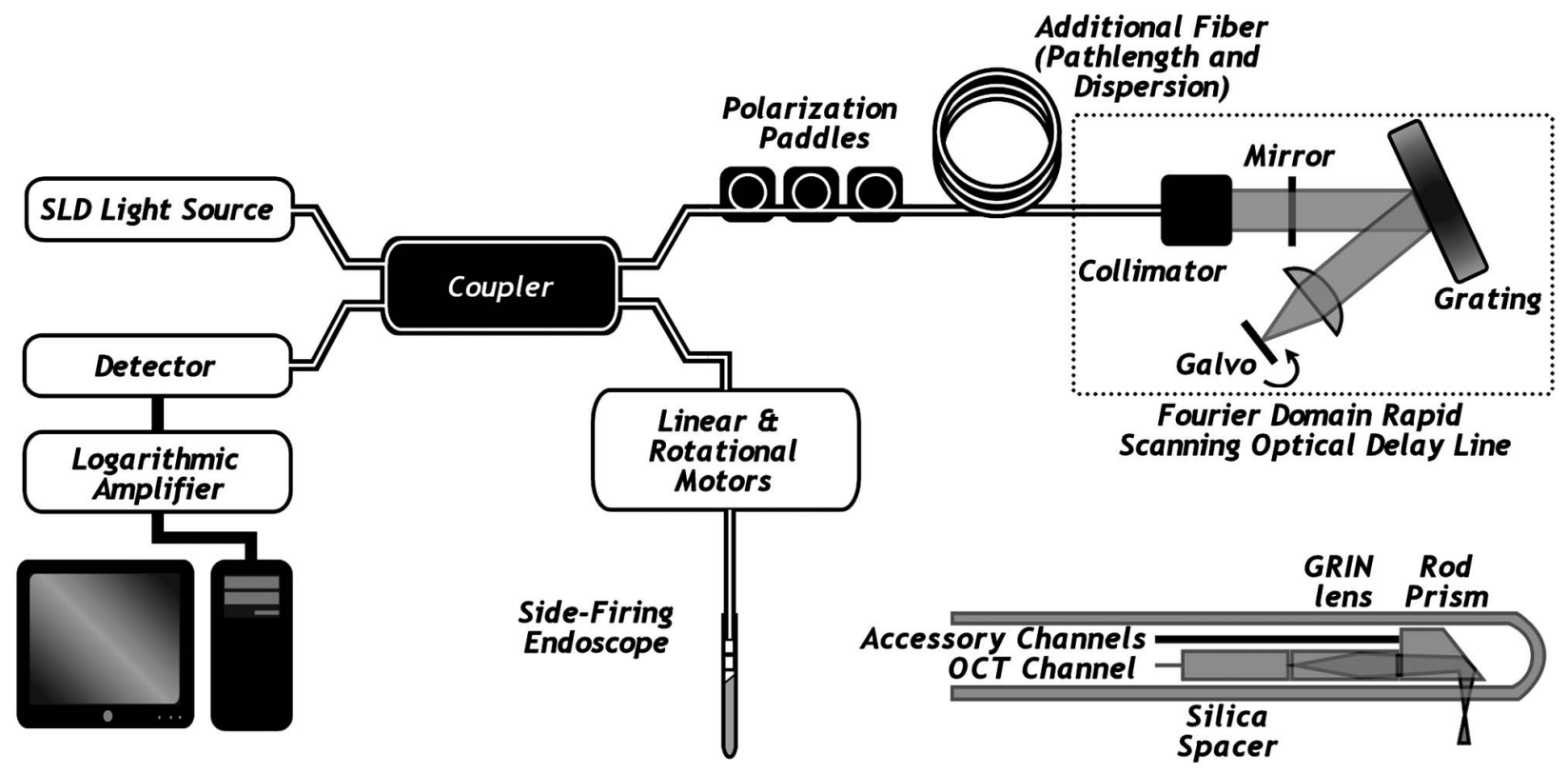

Schematic diagram of the endoscopic OCT system. 


\section{Figure 2}

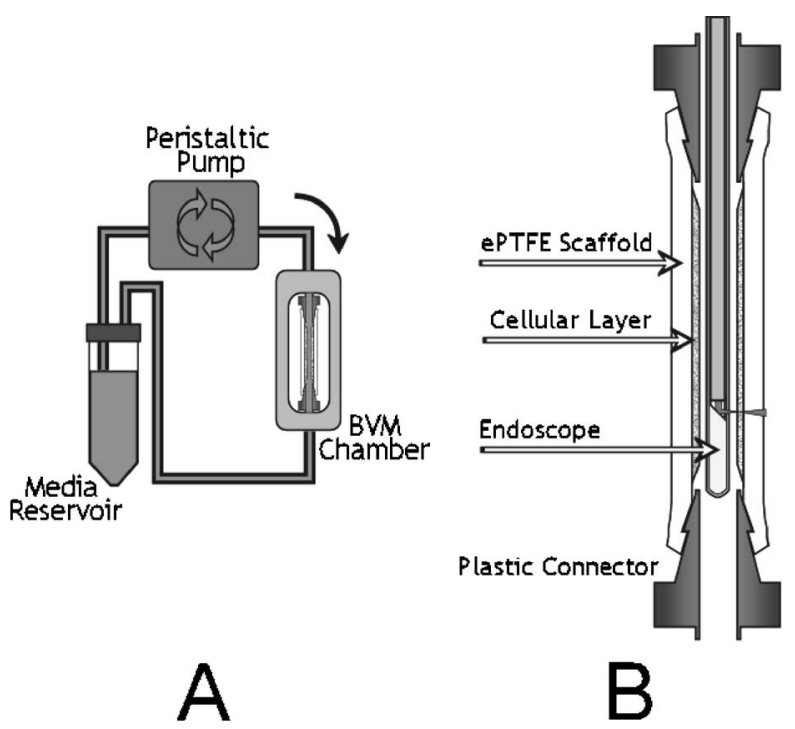

Fig. 2 Imaging of the BVM: (A) the bioreactor system and (B) schematic of BVM imaging geometry. 


\section{Figure 3}
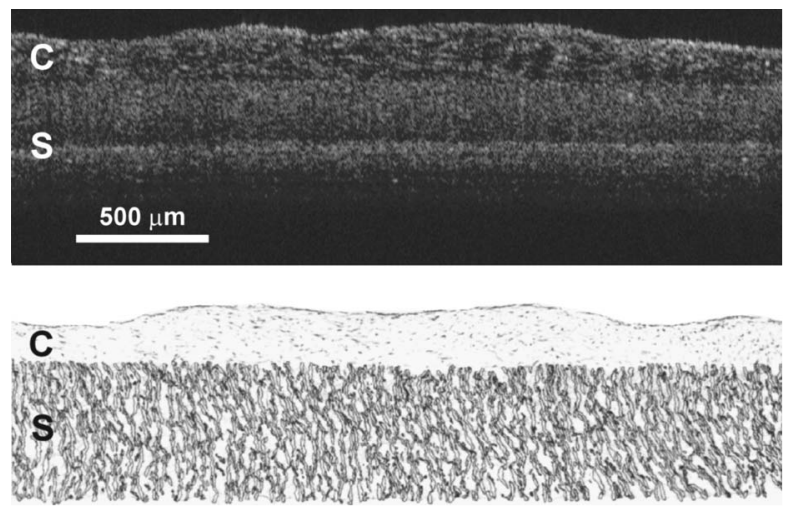

Fig. 3 Example comparison of OCT image (top) and light micrograph of an H\&E section (bottom) of the BVM; C, cell layer; S, scaffold. The bright horizontal feature in the scaffold is a reflection from the blue stripe in the scaffold material (not visible in histology image). Images are $0.96 \times 2.9 \mathrm{~mm}$. 


\section{Figure 4}
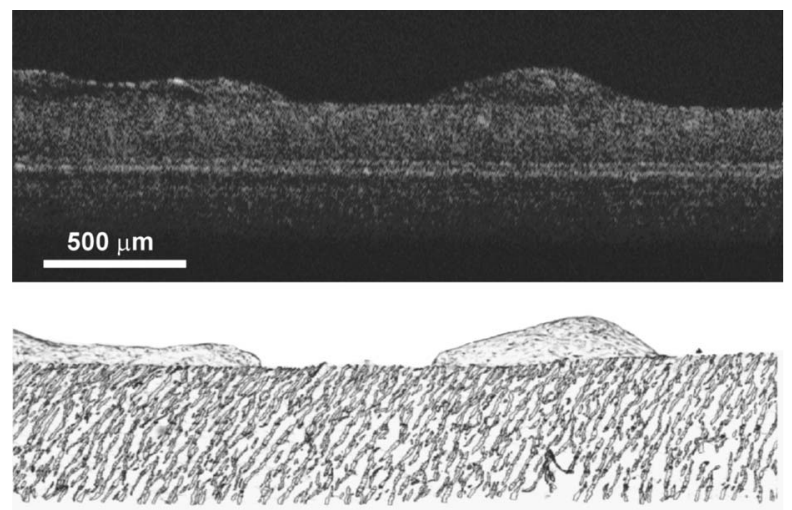

Fig. 4 Visualization of patches of cells with OCT and histology; example comparison of OCT image (top) and light micrograph of BVM histological section (bottom). Images are $0.96 \times 2.7 \mathrm{~mm}$. 


\section{Figure 5}

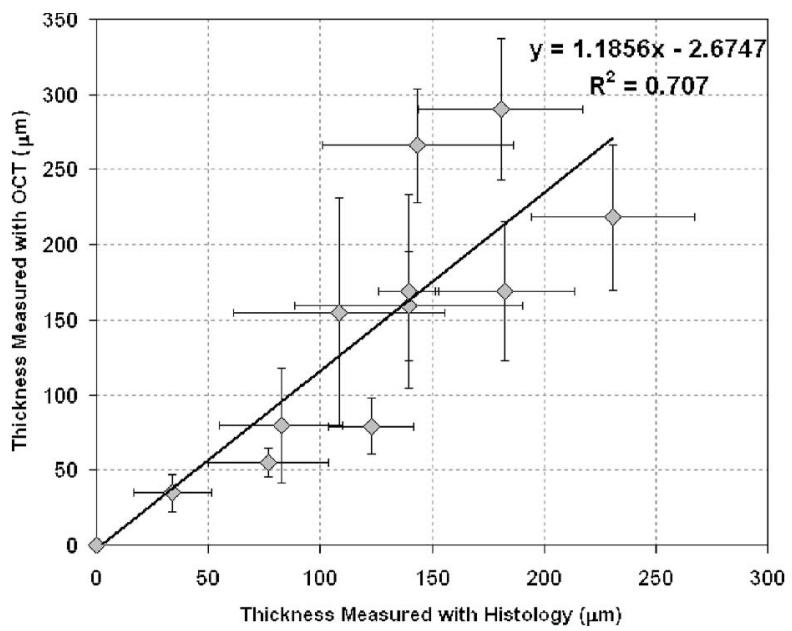

Fig. 5 Comparison of mean thickness measurements between OCT and the histological sections. The error bars are based on the standard deviation of the thickness measurements. 


\section{Figure 6}

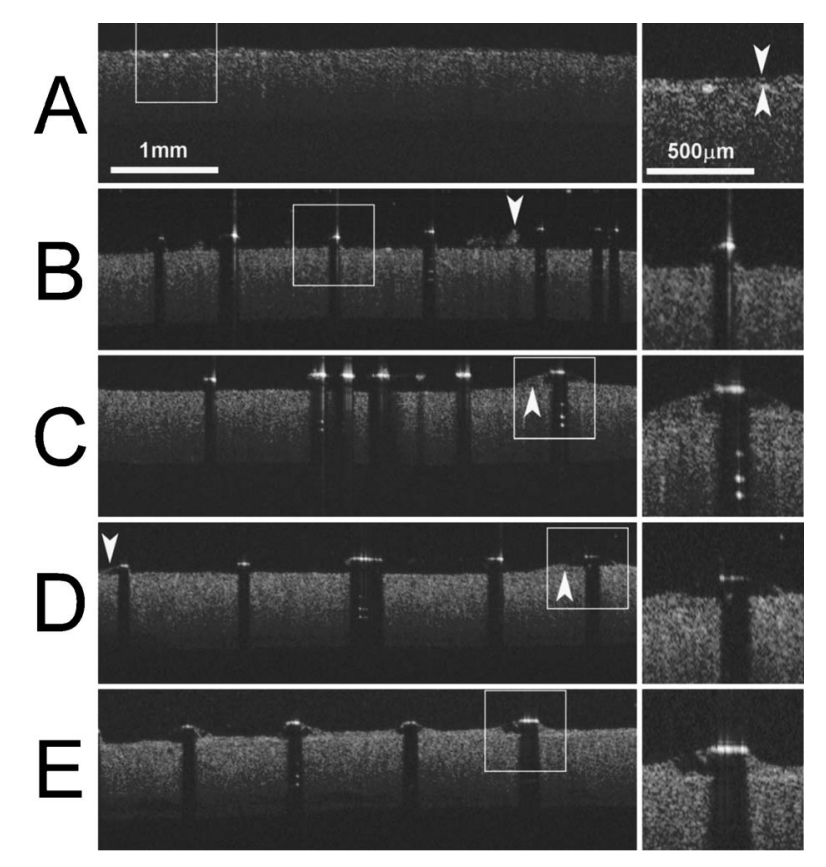

BVM response to bare metal stent: (A) a thin cellular lining was present before deployment; arrows delineate the thin hyperintense layer and (B) the postdeployment stent struts were visualized by a bright reflection followed by a dark vertical band. Disruption of cellular lining was visualized (arrows in B). Areas of cellular accumulation on the stent struts were seen in the 3- and 7-day images (arrows in $\mathrm{C}$ and D). Extensive cellular accumulation was observed at day 14 (E). 


\section{References}

1. M. E. Brezinski, "Optical coherence tomography for identifying unstable coronary plaque," Int. $J$. Cardiol. (2005).

2. M. E. Brezinski, G. J. Tearney, N. J. Weissman, S. A. Boppart, B. E. Bouma, M. R. Hee, A. E. Weyman, E. A. Swanson, J. F. Southern, and J. G. Fujimoto, "Assessing atherosclerotic plaque morphology: comparison of optical coherence tomography and high frequency intravascular ultrasound," Heart 77, 397-403 (1997).

3. T. Kume, T. Akasaka, T. Kawamoto, N. Watanabe, E. Toyota, Y. Neishi, R. Sukmawan, Y. Sadahira, and K. Yoshida, "Assessment of coronary intima - media thickness by optical coherence tomography: comparison with intravascular ultrasound," Jpn. Circ. J. 69, 903-907 (2005).

4. F. J. van der Meer, D. J. Faber, J. Perree, G. Pasterkamp, S. D. Baraznji, and T. G. van Leeuwen, "Quantitative optical coherence tomography of arterial wall components," Lasers Med. Sci. 20, 45-51 (2005).

5. M. E. Brezinski, G. J. Tearney, B. E. Bouma, J. A. Izatt, M. R. Hee, E. A. Swanson, J. F. Southern, and J. G. Fujimoto, "Optical coherence tomography for optical biopsy. Properties and demonstration of vascular pathology," Circulation 93, 1206-1213 (1996).

6. B. E. Bouma, G. J. Tearney, H. Yabushita, M. Shishkov, C. R. Kauffman, G. D. DeJoseph, B. D. MacNeill, S. L. Houser, H. T. Aretz, E. F. Halpern, and I. K. Jang, "Evaluation of intracoronary stenting by intravascular optical coherence tomography," Heart 89, 317-320, 2003).

7. L. J. Diaz-Sandoval, B. E. Bouma, G. J. Tearney, and I. K. Jang, "Optical coherence tomography as a tool for percutaneous coronary interventions," Catheter. Cardiovasc. Interv. 65, 492-496 (2005).

8. I. K. Jang, G. Tearney, and B. Bouma, "Visualization of tissue prolapse between coronary stent struts by optical coherence tomography: comparison with intravascular ultrasound," Circulation 104, 2754 (2001).

9. E. Regar, J. Schaar, and P. W. Serruys, "Images in cardiology. Acute recoil in sirolimus eluting stent: real time, in vivo assessment with optical coherence tomography," Heart 92, 123 (2006).

10. L. Buellesfeld, V. Lim, U. Gerckens, R. Mueller, and E. Grube, "Comparative endoluminal visualization of TAXUS crush-stenting at 9 months follow-up by intravascular ultrasound and optical coherence tomography," Z. Kardiol. 94, 690-694 (2005).

11. S. Ito, M. Itoh, and T. Suzuki, "Intracoronary imaging with optical coherence tomography after cutting balloon angioplasty for in-stent restenosis," J. Invasive. Cardiol. 17, 369-370 (2005).

12. T. Kume, T. Akasaka, T. Kawamoto, N. Watanabe, E. Toyota, R. Sukmawan, Y. Sadahira, and K. Yoshida, "Visualization of neointima formation by optical coherence tomography," Int. Heart J. 46, 1133- 1136 (2005).

13. M. Takano, I. K. Jang, and K. Mizuno, "Neointimal proliferation around malapposed struts of a sirolimus-eluting stent: optical coherence tomography findings," Eur. Heart J. 27, 1763 (2006).

14. J. Shite, D. Matsumoto, and M. Yokoyama, "Sirolimus-eluting stent fracture with thrombus, visualization by optical coherence tomography," Eur. Heart J. 27, 1389 (2005).

15. D. L. Fischman, M. B. Leon, D. S. Baim, R. A. Schatz, M. P. Savage, I. Penn, K. Detre, L. Veltri, D. Ricci, and M. Nobuyoshi, "A randomized comparison of coronary-stent placement and balloon angioplasty in the treatment of coronary artery disease. Stent Restenosis Study Investigators," $N$. Engl. J. Med. 331, 496-501 (1994).

16. P. W. Serruys, P. de Jaegere, F. Kiemeneij, C. Macaya, W. Rutsch, G. Heyndrickx, H. Emanuelsson, J. Marco, V. Legrand, and P. Materne, "A comparison of balloon-expandable-stent implantation with balloon angioplasty in patients with coronary artery disease. Benestent Study Group," $N$. Engl. J. Med. 331, 489-495 (1994).

17. A. M. Salam, J. Al Suwaidi, and D. R. Holmes, Jr., "Drug-eluting coronary stents," Curr. Probl Cardiol. 31, 8-119 (2006).

18. E. Van Belle, K. Abolmaali, C. Bauters, E. P. McFadden, J. M. La- blanche, and M. E. Bertrand, "Restenosis, late vessel occlusion and left ventricular function six months after balloon angioplasty in diabetic patients," J. Am. Coll. Cardiol. 34, 476-485 (1999). 
19. C. Di Mario, H. Griffiths, O. Goktekin, N. Peeters, J. Verbist, M. Bosiers, K. Deloose, B. Heublein, R. Rohde, V. Kasese, C. Ilsley, and R. Erbel, "Drug-eluting bioabsorbable magnesium stent," J. Interv. Cardiol. 17, 391-395 (2004).

20. H. Tamai, K. Igaki, E. Kyo, K. Kosuga, A. Kawashima, S. Matsui, H. Komori, T. Tsuji, S. Motohara, and H. Uehata, "Initial and 6-month results of biodegradable poly-1-lactic acid coronary stents in humans," Circulation 102, 399-404 (2000).

21. F. Vogt, A. Stein, G. Rettemeier, N. Krott, R. Hoffmann, D. J. vom, A. K. Bosserhoff, W. Michaeli, P. Hanrath, C. Weber, and R. Blindt, "Long-term assessment of a novel biodegradable paclitaxel-eluting coronary polylactide stent," Eur. Heart J. 25, 1330-1340 (2004).

22. K. O. Cardinal, G. T. Bonnema, H. Hofer, J. K. Barton, and S. K. Williams, "Tissue-engineered vascular grafts as in vitro blood vessel mimics for the evaluation of endothelialization of intravascular devices," Tissue Eng. 12, 3431-3438 (2006).

23. Y. Yang, A. Dubois, X. P. Qin, J. Li, A. E. Haj, and R. K. Wang, "Investigation of optical coherence tomography as an imaging modality in tissue engineering," Phys. Med. Biol. 51, 16491659 (2006).

24. J. K. Barton, D. B. Dal Ponte, S. K. Williams, B. Ford, and M. R. Descour, "Imaging vascular implants with optical coherence tomography," Proc. SPIE 3915, 229-236 (2000).

25. C. Mason, J. F. Markusen, M. A. Town, P. Dunnill, and R. K. Wang, "The potential of optical coherence tomography in the engineering of living tissue," Phys. Med. Biol. 49, 1097-1115 (2004).

26. A. M. Rollins, M. D. Kulkarni, S. Yazdanfar, R. Ung-arunyawee, and J. A. Izatt, "In vivo video rate optical coherence tomography," Opt. Express 3, 219-229 (1998).

27. A. R. Tumlinson, L. P. Hariri, U. Utzinger, and J. K. Barton, "Miniature endoscope for simultaneous optical coherence tomography and laser-induced fluorescence measurement," Appl. Opt. 43, 113-121 (2004).

28. R. Y. Kannan, H. J. Salacinski, P. E. Butler, G. Hamilton, and A. M. Seifalian, "Current status of prosthetic bypass grafts: a review," J. Biomed. Mater. Res., Part B: Appl. Biomater. 74, 570-581 (2005).

29. F. J. van der Meer, D. J. Faber, I. Cilesiz, M. J. C. van Germert, and T. G. van Leeuwen, "Temperature dependent optical properties of individual vascular wall componenets, measured by optical coherence tomography," J. Biomed. Opt. 11(4), 041120 (2006). 